\title{
OBESIDADE MÓRBIDA: controle dos riscos
}

DESCRITORES - Obesidade mórbida. Cirurgia bariátrica. Complicações pós-operatórias.

Os dados epidemiológicos referentes à obesidade têm se tornado alarmantes, sejam no que tangem às taxas crescentes de prevalência e incidência, sejam nas implicações relacionadas às doenças associadas, também conhecidas como co-morbidades. O panorama tem se apresentado cada vez mais crítico devido ao acometimento de população cada vez mais jovem, em especial a infanto-juvenil. Tal fato determina a ocorrência de doenças de caráter crônico já muito precocemente, acarretando uma série de disfunções com prejuízo das reservas clínicas, demandando tratamentos sucessivos com dispêndios progressivamente maiores e atingindo cifras inimagináveis ${ }^{(5)}$.

A preocupação com a obesidade tem assumido destaque no cenário internacional e nacional, haja vista os inúmeros fóruns e resoluções emanados dos principais órgãos de regulação da saúde, como a Organização Mundial de Saúde (OMS) e instituições administrativas, como o National Institute of Health (NIH) nos Estados Unidos e o Ministério da Saúde do Brasil ${ }^{(1)}$.

Dentre as várias formas de mensuração da obesidade, o índice de massa corpórea (IMC), desenvolvido pelo matemático belga Lambert Quetelet em meados do século XIX, tornou-se o padrão referencial para essa avaliação, tanto em âmbito individual como populacional ${ }^{(3)}$. Dada sua praticidade, simplicidade e elevado grau de confiabilidade na indicação da gordura corporal, correlacionando-se inclusive com medidas diretas, o IMC permitiu a classificação da obesidade em quatro níveis - graus I, II, III (grave ou mórbida) e super-obesidade, constituindo-se no mais importante índice, dentre vários outros parâmetros antropométricos e clínico-laboratoriais indicativos de obesidade e risco para doenças associadas.

A caracterização da gravidade da obesidade grau III (IMC maior de $40 \mathrm{~kg} / \mathrm{m}^{2}$ ) dá-se devido à conjunção de três aspectos: prevalência elevada da compulsão alimentar, resistência aos tratamentos clínicos (perda de peso insuficiente ou não sustentada) e associação freqüente com doenças inter-relacionadas, que são provocadas ou agravadas pela obesidade e que melhoram com a redução e controle do peso. Dentre as principais comorbidades destacam-se a diabetes, hipertensão arterial e as dislipidemias, que constituem um conjunto de fatores de risco cardiovascular, relacionados especialmente à deposição de gordura visceral e resistência à insulina, denominado de síndrome metabólica.

O tratamento cirúrgico da obesidade, com indicações já bem estabelecidas nas formas graves ou intermediarias com doenças associadas relevantes, tem proporcionado excelentes resultados no que concerne à manutenção da perda de peso em níveis aceitáveis. Mesmo dentre aqueles que têm re-ganhado uma fração de peso, a maioria não volta a atingir os níveis prévios de morbidez.

O recente avanço no campo dos enterohormônios tem promovido mudança no panorama de interpretação de funcionamento das várias técnicas cirúrgicas existentes, determinando modificações e surgimento de novas propostas, apoiadas fundamentalmente no controle do binômio fome-saciedade e na regulação do metabolismo da insulina.

O procedimento cirúrgico mais comumente utilizado é a gastroplastia com derivação em Y-de-Roux, conhecida em nosso meio como operação de Fobi-Capella, independentemente da colocação ou não de anel constritor ao nível da bolsa gástrica. Tal nominação decorre da ampla divulgação deste procedimento a partir de sua utilização pioneira na Disciplina de Cirurgia do Aparelho Digestivo do Hospital das Clínicas da Faculdade de Medicina da Universidade de São Paulo, no início dos anos 90, com os trabalhos de GARRIDO Jr. ${ }^{(2)}$.

Importante linha de pesquisa que tem se estabelecido, principalmente nos centros de referência, é a identificação de fatores de predição de resultados, sejam clínicos ou cirúrgicos.

No artigo publicado neste número dos ARQUIVOS de GASTROENTEROLOGIA ${ }^{(4)}$, oriundo de um dos principais centros de cirurgia bariátrica do país, objetiva-se a identificação de fatores de risco para complicações pós-operatórias. Destacase a maior relevância do trabalho se considerarmos, ainda, a alta prevalência da obesidade em níveis mais elevados, super-obesidade, nos serviços públicos de atendimento. Numa ampla análise estatística multivariada, conclui-se que o IMC acima de $55 \mathrm{~kg} / \mathrm{m}^{2}$ constitui o principal fator de risco para complicações graves e óbito. Em consonância com vários trabalhos da literatura, discutem-se aspectos relacionados às principais complicações no período pósoperatório precoce. Por fim, destaca-se a importância de adequado preparo pré-operatório e de cuidados no período intra e pós-operatório imediato. 
Estes dados reforçam de forma objetiva a necessidade de preparação pré-operatória neste grupo especial de pacientes super-obesos, que implica na avaliação clínica e detecção de repercussões funcionais, especialmente as de natureza cardiorespiratória. Ressalta-se a importância de medidas para favorecer a perda de peso na fase pré-operatória, da ordem de $10 \%$, com intuito de minimizar a ocorrência de complicações pós-operatórias.

Nesta mesma vertente, a Unidade de Cirurgia Bariátrica e Metabólica do Hospital das Clínicas da Faculdade de Medicina da Universidade de São Paulo tem promovido uma associação com o Hospital de Retaguarda de Suzano, SP, para internação de pacientes super-obesos para submeterem-se a tratamento dietético com vistas à perda de peso no preparo pré-operatório.

Por fim, vale destacar a importância do estabelecimento de centros especializados no tratamento da obesidade mórbida, uma vez que sob este manto reúnem-se múltiplas faces de uma afecção complexa e extremamente grave.

\section{Marco Aurelio SANTO ${ }^{1}$ Ivan CECCONELLO ${ }^{2}$}

Santo MA, Cecconello I. Morbid obesity: risks control. Arq Gastroenterol. 2008;45(1):1-2.

HEADINGS - Obesity, morbid. Bariatric surgery. Postoperative complications.

\section{REFERÊNCIAS}

1. Brasil. Ministério da Saúde. Secretaria de Assistência à Saúde. Portaria n. 492, de 31 de agosto de 2007. Definir Unidade de Assistência de Alta Complexidade ao Paciente Portador de Obesidade Grave como o hospital que ofereça assistência diagnóstica e terapêutica especializada, de média e alta complexidade, condições técnicas, instalações físicas, equipamentos e recursos humanos adequados ao atendimento às pessoas portadoras de obesidade grave. Diário Oficial da União, Brasilia (DF). 200731 ago; Seção I:31. Disponível em: http://dtr2001.saude.gov.br/sas/PORTARIAS/Port2007/PT-492.htm.
2. Garrido Jr AB, editor. Cirurgia da obesidade. São Paulo: Atheneu; 2002.

3. Keil U, Kuulasmaa K. WHO MONICA Project risk factors. Int J Epidemiol. 1989;18 (Suppl 1):s46-s55.

4. Martins-Filho ED, Câmara-Neto JB, Bandeira-Ferraz AA, Amorim M, MachadoFerraz E. Evaluation of risk factors in superobese patients submitted to conventional Fobi-Capella surgery. Arq Gastroenterol. 2008;45(1):3-10.

5. Obesity: preventing and managing the global epidemic. Report of a WHO Consultation World Health Organization Tech Rep Ser. 2000;894:i-xii, 1-253.

`Unidade de Cirurgia Bariátrica e Metabólica do Hospital das Clínicas da Faculdade de Medicina da Universidade de São Paulo.

2Disciplinas de Cirurgia do Aparelho Digestivo e Coloproctologia da Faculdade de Medicina da Universidade de São Paulo. 